\title{
Evidence for the Tunneling Site on Transition-Metal Oxides: $\mathrm{TiO}_{2}(110)$
}

\author{
Ulrike Diebold and J.F. Anderson \\ Department of Physics, Tulane University, New Orleans, Louisiana 70118 \\ Kwok-On Ng and David Vanderbilt \\ Department of Physics and Astronomy and Laboratory for Surface Modification, Rutgers, \\ The State University of New Jersey, Piscataway, New Jersey 08904
}

(Received 14 March 1996)

\begin{abstract}
We present atomically resolved scanning tunneling microscopy (STM) images from $\mathrm{TiO}_{2}(110)$ surfaces. After annealing nearly perfect stoichiometric $1 \times 1$ surfaces to elevated temperatures in ultrahigh vacuum, randomly distributed oxygen vacancies are observed. The apparent shape of these defects provides strong evidence that the STM is imaging undercoordinated Ti atoms, as do firstprinciples pseudopotential calculations of the electronic states accessible to tunneling. We thus resolve a controversy as to whether STM imaging on this surface is dominated by geometric-structure or electronic effects. [S0031-9007(96)00905-2]
\end{abstract}

PACS numbers: 68.35.Bs, 61.16.Ch, 61.72.Ji, 68.35.Dv

Transition-metal oxides play an important role in a wide range of applications, and their surfaces crucially influence the behavior of gas sensors, catalysts, semiconductor devices, and environmental degradation processes. The desire to improve materials applications motivates the increased efforts to understand the structure and chemistry of single-crystalline transition-metal oxide surfaces at an atomic level. Much progress has been made in recent years [1], but many fundamental questions are still open. For example, defects strongly affect or even dominate the chemical and electronic properties of oxide surfaces, but the exact geometry of these defects is hardly investigated so far. In this context, the rutile $\mathrm{TiO}_{2}(110)$ surface has emerged as one of the most important model systems for oxide surfaces. Titanium dioxide has important applications in catalysis and photocatalysis, and its surfaces have been the subject of numerous surface science investigations (see Henrich and Cox [1], and references therein). While there is ample experimental [2-6] and theoretical $[7,8]$ evidence that a simple charge-neutral truncation is the correct structure for the stoichiometric $(110)-(1 \times 1)$ surface, several more complex structures have been observed after high-temperature annealing [2-6,9], and their interpretation has remained controversial.

Naturally, scanning tunneling microscopy (STM) is an extremely promising tool for studying atomic-scale structure on these surfaces. However, the interpretation of STM images on transition-metal oxide surfaces is often severely hampered by uncertainty as to whether the image contrast is governed by geometric or electronic-structure effects. For $\mathrm{TiO}_{2}$, for example, atomic-resolution STM is only successful when imaging unoccupied states (positive bias) on slightly reduced (n-type) samples. For such samples, the Fermi level is pinned at the conduction band minimum $(\mathrm{CBM})$ in the $3 \mathrm{eV}$ gap, so that the electrons are much more mobile than the holes. Under a typical bias of $+2 \mathrm{~V}$, electrons can thus tunnel from the tip into states within $\sim 2 \mathrm{eV}$ above the CBM and be conducted away from the surface. On the one hand, these CBM states have primarily cation $d$ character (the valence band having primarily $\mathrm{O} 2 p$ character) [10], so that one might expect to image the metal atoms as the "white" features in STM topographs. On the other hand, the oxygen atoms are most exposed on many oxide surfaces, and it seems plausible that geometrical considerations might dominate. On the (110) face of $\mathrm{TiO}_{2}$, Fig. 1 (inset) shows that the physical topography is strongly dominated by protruding rows of bridging oxygen atoms. Thus, a controversy has arisen as to whether to interpret the white rows of the STM topographs as geometrical (oxygen) features $[2,4]$ or electronic-structure (Ti) features $[2,3,5,6]$.

Here, we use STM studies of single-surface oxygen vacancies, together with theoretical calculations of the surface electronic structure, to resolve this question, at least for $\mathrm{TiO}_{2}(110)$. From the position of the oxygen vacancies relative to the white rows, we distinguish between the two cases. By analyzing the local electron density of states that contributes to the STM current, we identify the tunneling sites. Both the experimental results and the theoretical analysis lead to the conclusion that the electronic-structure features dominate the STM images of this transition-metal oxide.

Our theoretical analysis is based on first-principles plane-wave pseudopotential calculations carried out within the local-density approximation (LDA) following the methods of Ref. [7]. The pseudopotentials for Ti and $\mathrm{O}$ are those used in Ref. [7] and were generated using an ultrasoft pseudopotential scheme [11]. Periodic supercells containing 18 and 30 atoms were used to study the stoichiometric $1 \times 1$ surface, while a 34-atom cell was used for the nonstoichiometric $2 \times 1$ surface. For both cases, special $k$-point sets were chosen to correspond to a 16-point set in the full Brillouin zone of the $1 \times 1$ surface. Self-consistent total-energy and force 


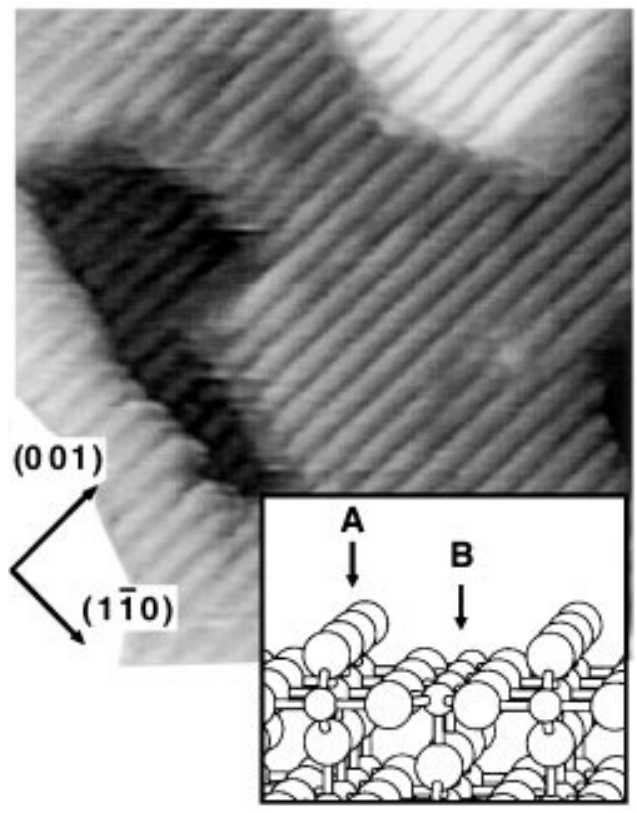

FIG. 1. STM image of a stoichiometric $1 \times 1 \quad \mathrm{TiO}_{2}(110)$ surface, $140 \times 140 \AA^{2}$. Sample bias $+1.6 \mathrm{~V}$, tunneling current $0.38 \mathrm{nA}$. The inset shows a ball-and-stick model of the unrelaxed $1 \times 1 \mathrm{TiO}_{2}(110)$ surface. Rows of bridging oxygen atoms are labeled " $A$," rows of fivefold coordinated titaniums " $B$. ."

calculations were used to relax the atomic coordinates until the forces were less that $0.1 \mathrm{eV} / \AA$. The relaxed structure has been described and discussed in Ref. [7]. A band-structure run was carried out to obtain the valenceand conduction-band electronic wave functions. These were used to extract information about the local density of states above the surface for comparison with the STM studies, as will be discussed below.

The experiments were performed in an ultrahighvacuum (UHV) chamber with an Omicron STM1 scanning tunneling microscope operated at room temperature, using etched $\mathrm{W}$ tips. The images shown are constant-current topographs taken at positive sample bias (imaging unoccupied states). For stoichiometric $\mathrm{TiO}_{2}$ surfaces, no stable imaging was possible for negative sample bias voltages as is also reported by other authors $[2,3,5,6]$. The $\mathrm{TiO}_{2}$ crystal (Commercial Crystal Laboratories) was mounted on a Ta holder, and the sample was radiatively heated from the back through a hole in the holder. Charging problems with $\mathrm{TiO}_{2}$ single crystals can be avoided by a bulk reduction via an initial high-temperature anneal. This consisted of annealing the fresh sample to $870 \mathrm{~K}$ for $3 \mathrm{~h}$, resulting in a bluish sample color and $n$-type semiconducting bulk properties. Sputtering was performed using $1 \mathrm{keV} \mathrm{Ar}^{+}$ ions with a typical sample current density of $8 \mu \mathrm{A} / \mathrm{cm}^{2}$. $\mathrm{X}$-ray photoelectron spectroscopy (XPS) was used for checking sample cleanliness and oxygen reduction; the shape of the Ti $2 p$ peak is quite sensitive to the level of stoichiometry [12]. Ca segregated to the surface initially, but disappeared after three sputtering-annealing cycles.

We find that the exact annealing procedure is important for the degree of surface quality. The cleaning recipes described in detail by Pan et al. [12] are followed. The stoichiometric $\mathrm{TiO}_{2}$ surface was prepared by slowly increasing the sample temperature in $2 \times 10^{-6}$ mbar oxygen to $700 \mathrm{~K}$, holding this temperature for at least 10 min, slowly cooling the sample to room temperature and waiting approximately 30 more minutes before reducing the oxygen background pressure. According to XPS, this procedure results in a stoichiometric surface. However, a second annealing to $550 \mathrm{~K}$ was necessary before stable, high-quality STM images could be obtained. Possibly this last step removed residual impurity adsorbates (e.g., $\mathrm{OH})$ that had formed on the surface during the prolonged oxygen exposure.

Figure 1 shows an STM image of a stoichiometric surface obtained by this procedure; nearly perfect shortrange order is achieved. The average terrace size is relatively small $(\sim 100 \AA)$, and a step height of $3.2 \AA$ is observed in agreement with the model depicted in Fig. 1. The image shown is typical for a well-prepared surface; images of comparable quality were obtained on many different areas of the crystal. Bright and dark rows run along the [001] direction in Fig. 1. The distance between the rows is $6.3 \pm 0.25 \AA$, in agreement with the unit cell dimension of $6.5 \AA$ along [110], and the observed corrugation along this direction is $1.2 \AA$. As outlined in the introduction of this paper, it is not immediately obvious if the bright rows correspond to lines of "bridging oxygen" atoms (labeled " $A$ " in the inset of Fig. 1) or fivefold coordinated $\mathrm{Ti}^{4+}$ ions (" $B$ "). The bridging oxygen rows protrude from the surface plane by $1.13 \AA$ on a relaxed $\mathrm{TiO}_{2}(110)$ surface [7], so if STM were dominated by topographical effects, they would appear as rows with high contrast in Fig. 1. As we shall see, this is not the case.

We used the pseudopotential calculations to analyze the local density of states in the vacuum region above the surface. In rough correspondence with the experimental bias conditions, we summed the charge density of conductionband states from 0 to $2 \mathrm{eV}$ above the conduction-band minimum [13]. This quantity was then averaged over the [001] direction and plotted as a function of the other two coordinates, as shown in Fig. 2(a). The plot shown is for a relaxed 18-atom slab, but calculations for a thicker 30 -atom slab gave very similar results. Under constantcurrent tunneling conditions, the STM tip is roughly expected to follow one of the equal-density contours several angstroms above the surface. The plot in Fig. 2(a) clearly shows that the charge-density contours extend higher above the fivefold coordinated Ti atoms, in spite of the physical protrusion of the bridging oxygen atoms. This confirms that the STM is imaging the surface Ti atoms, i.e., that the apparent corrugation is reversed from 
(a)

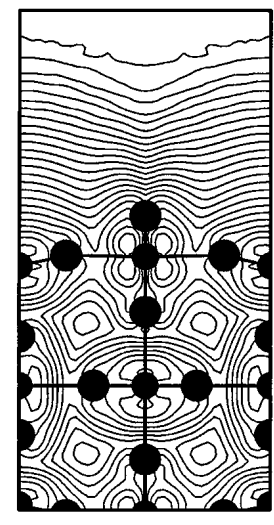

(b)

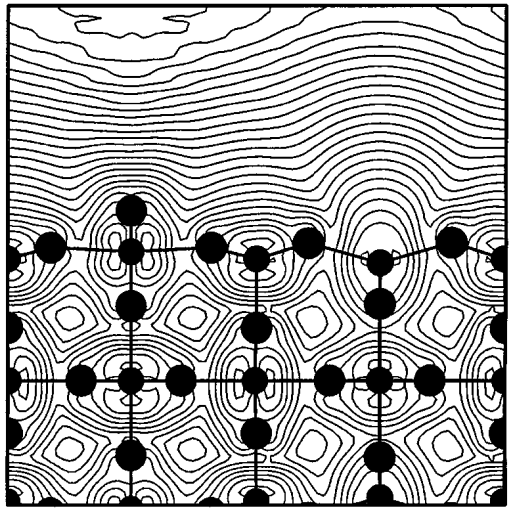

FIG. 2. Contour plots of [001]-averaged charge densities associated with electron states within $2 \mathrm{eV}$ of the conduction band minimum for (a) the relaxed stoichiometric $1 \times 1$ surface and (b) the relaxed oxygen-deficient $2 \times 1$ surface. Contour levels correspond to a geometric progression of charge density, with a factor of 0.56 separating neighboring contours. The "noise" in the topmost contours is an artifact of the periodically repeated slab geometry.

naïve expectations by electronic-structure effects. The apparent corrugation at a distance of 4-5 $\AA$ above the surface is about $0.5-0.6 \AA$, in reasonable agreement with the experimentally observed one.

After annealing stoichiometric surfaces to temperatures above $700 \mathrm{~K}$ in ultrahigh vacuum, new features are seen in the STM images. They consist of short, bright rows in the [1] 0$]$ direction connecting two [001]-oriented bright rows as shown Fig. 3 and its inset. We assign these features as single missing oxygen atoms (see below). The oxygen vacancies are randomly distributed on terraces, with nucleation along the step edges running parallel to [001] (not shown). The three brightest features in the image are presumably related to impurities, and are not of further interest here. The defect concentration has been evaluated from numerous images from samples with annealing times varying between $10 \mathrm{~min}$ and $2 \mathrm{~h}$. The concentration of missing oxygen atoms was determined

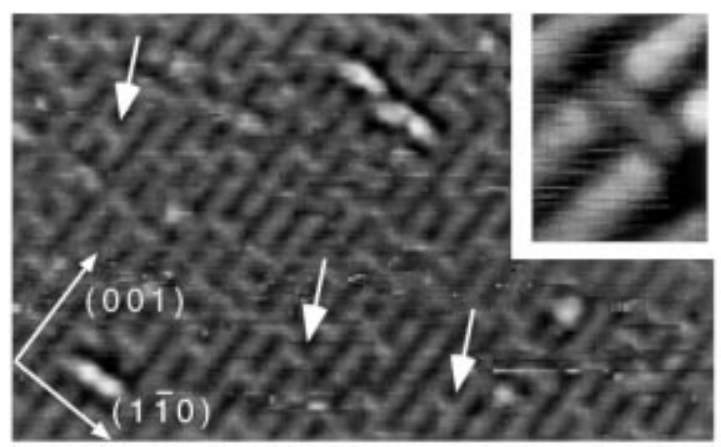

FIG. 3. STM image of a slightly oxygen-deficient $\mathrm{TiO}_{2}(110)$ surface, $190 \times 120 \AA^{2}$. The arrows point to the features assigned as single oxygen vacancies, one of which is enlarged in the inset. Sample bias $+1.2 \mathrm{~V}$, tunneling current $0.5 \mathrm{nA}$. from the number of defect features on STM images divided by the number of oxygen atoms expected from the $(1 \times 1)$ surface structure. Between $2 \%$ and $5 \%$ of missing oxygen atoms are found, with somewhat higher fractions for longer annealing times and temperatures. This is of the same order of magnitude as found in a lowenergy ion scattering experiment where missing oxygen ions were replaced by absorbing ${ }^{18} \mathrm{O}$ on slightly defective, vacuum-annealed surfaces [12].

It is well established that annealing to elevated temperatures creates oxygen-deficient, reduced $\mathrm{TiO}_{2}(110)$ surfaces [1]. If neutral $\mathrm{O}$ atoms are removed from the surface, an electron pair is left behind which reduces the charge state of neighboring Ti ions, as is observed clearly in photoemission experiments [1]. Removal of twofold coordinated bridging oxygen atoms is more likely than removal of threefold coordinated in-plane oxygens, although so far there is no direct experimental evidence for a propensity of one over the other. Let us assume that a bridging oxygen is removed upon point defect formation in our case. Then, the defect would appear as a bright feature on the dark rows if the unoccupied density of states concentrated on the fivefold coordinated $\mathrm{Ti}$ were the main cause for image contrast in STM images. The same defect would appear as a dark spot on a bright row if the bridging oxygens were imaged. In our images, the latter is never observed [14], which implies that indeed the fivefold coordinated Ti rows are imaged and that the observed STM topography is reversed with respect to the physical topography. Removal of an in-plane oxygen is expected to cause a much more asymmetric feature than the one observed. Relatively minor relaxations of atomic positions have been calculated upon removal of bridging oxygen atoms on $\mathrm{TiO}_{2}(110)$ [7].

Because of the very large supercell that would be needed, it was not practical to apply the pseudopotential calculations to the case of an isolated oxygen vacancy. However, our conclusions are supported by a calculation on a $2 \times 1$ structure consisting of alternate missing rows of bridging oxygen atoms, as shown in Fig. 2(b). The slab thickness and other theoretical details are the same as for Fig. 2(a). A sharp increase in conduction-band charge density in the vicinity of the missing bridging $\mathrm{O}$ atoms, and a depletion of charge around the remaining ones, is clearly evident. Again, one finds that the apparent corrugation is the reverse of the geometric one.

Our conclusion that fivefold $\mathrm{Ti}$ atoms are imaged is in agreement with the STM observation that formate ions are adsorbed on the bright rows [5], and with the fact that contrast reversal is observed for dilute $\mathrm{O}$ atoms adsorbed on $\mathrm{Ti}(0001)$ faces when the gap voltage polarity is switched [15]. However, it is in disagreement with the interpretation of defects given by Fischer et al. [4]. We suspect this discrepancy might be due to different sample preparation procedures. In view of the demonstration that single oxygen vacancies can be prepared by starting from 
a well-defined, stoichiometric surface with subsequent gentle annealing in UHV to control the defect density $[12,16]$, we have taken particular care to follow this recipe closely. Judging from the high quality of our stoichiometric surfaces (Fig. 1), we infer that the observed features are due to oxygen vacancies and not due to surface impurities.

The confirmation that the contrast in positive samplebias STM images is due to $\mathrm{Ti}$ atoms rather than bridging oxygens has interesting consequences for the interpretation of other structures observed in STM images. Several authors report single line defects appearing as extra-bright rows on the $(1 \times 1)$ surface [2-5]. These "double-strand" rows, which have been assigned as precursors to the fully $(1 \times 2)$ reconstructed surface, occur in registry with the bright rows on the $(1 \times 1)$ surface, hence at the position of fivefold coordinated $\mathrm{Ti}$ atoms. Consequently, these line defects cannot be caused by a simple removal of one bridging oxygen row, as has been considered as a possibility [2,3,6,9]. Fischer et al. proposed that not only the bridging oxygens are removed but also the $\mathrm{Ti}$ atoms located underneath [4]; one would expect a massive reconstruction of the surroundings upon removal of so many atoms. Onishi et al. [5] proposed an alternative model, where bright line defects are due to an added double row of $\mathrm{Ti}_{2} \mathrm{O}_{3}$ units; this model is in agreement with our results. As pointed out above, the single line defects are probably the first stage of the often observed $(1 \times 2)$ reconstruction. While it has been assumed by most authors that this reconstruction is due to removal of every other bridging oxygen row $[3,6,9]$, it appears that this interpretation for the $1 \times 2$ surface has to be reconsidered.

In summary, we have shown that the electronic structure, rather than the physical topography, is the dominant factor for the contrast in STM images from a $1 \times 1 \mathrm{TiO}_{2}(110)$ surface. While we cannot be certain that the present results will generalize to other transitionmetal surfaces, the present work makes it more likely that electronic-structure effects will dominate over geometrical ones in these systems as well.

This work was supported, in part, by LEQSF(199497)-RD-A-26, the Petroleum Research Fund, the Center for Photoinduced Processes (NSF-EPSCoR), and DoEEPSCoR. K.-O.N. and D.V. acknowledge support by
NSF Grant DMR-91-15342. The authors wish to thank Theordore E. Madey for useful discussions.

[1] V.E. Henrich and P. A. Cox, The Surface Science of Metal Oxides (Cambridge University Press, Cambridge, 1994).

[2] D. Novak, E. Garfunkel, and T. Gustafsson, Phys. Rev. B 50, 5000 (1994).

[3] P. W. Murray, N. G. Condon, and G. Thornton, Phys. Rev. B 51, 10989 (1995).

[4] S. Fischer, A. W. Munz, K.-D. Schierbaum, and W. Göpel, Surf. Sci. 337, 17 (1995).

[5] H. Onishi, and Y. Iwasawa, Surf. Sci. 313, L783 (1994); H. Onishi, K. Fukui, and Y. Iwasawa, Bull. Chem. Soc. Jpn. 68, 2447 (1995); H. Onishi and Y. Iwasawa, Chem. Phys. Lett. 226, 111 (1994); H. Onishi and Y. Iwasawa, Phys. Rev. Lett. 76, 791 (1996).

[6] A. Szabo and T. Engel, Surf. Sci. 329, 241 (1995); M. Sander and T. Engel, Surf. Sci. Lett. 302, L263 (1994).

[7] M. Ramamoorthy, R. D. King-Smith, and D. Vanderbilt, Phys. Rev. B 49, 7709 (1994); M. Ramamoorthy, D. Vanderbilt, and R. D. King-Smith, Phys. Rev. B 49, 16721 (1994).

[8] P. Reinhardt and B. A. Hess, Phys. Rev. B 50, 12015 (1994); J. Goniakowski et al., Phys. Rev. B 53, 957 (1996).

[9] P. J. Møller and M.-C. Wu, Surf. Sci. 224, 265 (1989).

[10] K. M. Glassford and J. R. Cheilkowsky, Phys. Rev. B 46, 1284 (1992).

[11] D. Vanderbilt, Phys. Rev. B 41, 7892 (1990).

[12] J.-M. Pan, B. L. Maschhoff, U. Diebold, and T. E. Madey, J. Vac. Sci. Technol. A 10, 2470 (1992).

[13] For technical reasons, core augmentation charges associated with the ultrasoft pseudopotentials were not included. This omission has no effect on the charge density in the vacuum region of interest here.

[14] The observed shape of the defect itself is quite interesting; the two titanium atoms next to the missing $\mathrm{O}$ atom appear to be contracted towards the defect and somewhat darker. This may be caused by relaxations, or by hybridization between the in-plane $\mathrm{Ti}$ and the titaniums exposed by oxygen removal.

[15] R. Berndt, J. K. Gimzewski, and R. R. Schlitter, Surf. Sci. 310, 85 (1994).

[16] G. Lu, A. Linsebigler, and J. T. Yates, Jr., J. Phys. Chem. 98, 1173 (1994). 